\title{
HYDROGEN PEROXIDE INDUCED OXIDATIVE DAMAGE ON MECHANICAL PROPERTIES OF THE ARTICULAR CARTILAGE
}

\author{
EKREM CICEK* \\ Department of Physics, Faculty of Science \& Art, Mehmet Akif Ersoy University, Burdur, Turkey
}

(Received: July 21, 2017; accepted: October 3, 2017)

\begin{abstract}
Articular cartilage has unique mechanical and physicochemical properties which are responsible for its load carrying capabilities. This work investigates the effects of hydrogen peroxide induced oxidative damage on mechanical properties of articular cartilage. Bovine articular cartilage was exposed to hydrogen peroxide for a week. Dynamic and static mechanical tests applied to calculate articular cartilage compressive modulus. We observed higher control curve slopes than that of hydrogen peroxide curves which account for lesser stiffness values in the exposed articular cartilage. For the instantaneous experiments, results were statistically significant $(p=0.01, p<0.05)$. Here report that hydrogen peroxide induced oxidative damage causes reduction in the stiffness of the articular cartilage.
\end{abstract}

Keywords: Antiseptic - articular cartilage - compressive modulus - hydrogen peroxide

\section{INTRODUCTION}

Articular cartilage is an avascular connective tissue with remarkable biomechanical properties. The function of articular cartilage is reducing the friction among bones in joints via joint lubrication and smoothness of surfaces, distributing loads across the joint surface and shock absorption $[4,18]$. The main components of extracellular matrix are aggrecan and type II collagen which are most notably responsible for cartilage compressive and tensile resistance [22].

The mechanical properties of articular cartilage depend on the interaction among the chondrocytes and the matrix $[4,10]$. Impact of mechanical loading can lead to degradation of cartilage matrix cause in impaired functional properties of the cartilage $[11,16]$.

As it can kill anaerobic bacteria, hydrogen peroxide $\left(\mathrm{H}_{2} \mathrm{O}_{2}\right)$ is widely used for the prevention of local bacterial infection and clinical debridement in bone and joint surgeries $[1,7,19]$. However, the treatment of local wounds with antiseptics such as hydrogen peroxide induces tissue toxicity through intensive oxidation $[20,21]$.

\footnotetext{
*Corresponding author; e-mail address: ekrcicek@gmail.com
} 
Cartilage damaged by hydrogen peroxide has a limited capacity to regenerate and chondrocyte recovery is very difficult once the cartilage is damaged $[1,7,19]$.

Elevated reactive oxygen species (ROS) production in cartilage tissue, which is an immediate effect of hydrogen peroxide, can cause chondrocyte apoptosis and articular cartilage degradation. Hence, it can contribute to the aging of cartilage and the pathogenesis of osteoarthritis [17].

Although there are many studies related to various effects of hydrogen peroxide on articular cartilage, hydrogen peroxide induced oxidative damage on biomechanical properties of the articular cartilage have not been subjected to detailed studies [20]. There is a tradeoff between elimination of bacteria and toxicity to cartilage in this practice $[20,21]$. In this study, we investigated the immediate toxic cartilage damage after treatment with hydrogen peroxide. Dynamic (instantaneous) and static (equilibrium) mechanical tests were applied to calculate compressive modulus in articular cartilage.

\section{MATERIALS AND METHODS}

Articular cartilage samples were taken from mature bovine patellae obtained from a local abattoir on the same day after slaughter. To avoid any physical changes, the samples were kept and brought to lab via flasks. In the laboratory, after removing the surrounding soft tissue, osteochondral explants $(\sim 5 \times 5 \mathrm{~mm})$ were harvested from the lateral tibial plateau and transported in phosphate-buffered saline (PBS). Before compression tests, cartilage samples were placed to $30 \% \mathrm{H}_{2} \mathrm{O}_{2}(300 \mathrm{ml})$ for a week, to simulate the long-term exposure under in vitro conditions rather than instant effects of the standard clinical application of 3\%, and the control samples were soaked with phosphate buffered saline solution for the same period with experimental group. Two different mechanical tests, instantaneous and equilibrium, were applied to calculate compressive modulus for articular cartilage.

\section{Compression tests for instantaneous modulus}

With the aim of establishing the operability and technical parameters, the device was subjected to comparative test trials. For compression, a LR5K Plus universal tensile testing machine and a homemade compression holder having a diameter of $3 \mathrm{~cm}$ was clamped to it were used.

The total number of test samples subjected to compression is 25 , composed of 7 control and 18 hydrogen peroxide exposed experimental group samples.

The samples were placed in the designed tester device and the resulting diagrams were obtained as shown in Fig. 1 for the LR5K Plus tester. The test results were drawn as diagrams in NEXYGEN Plus Data Analysis Software and MS Excel 2010. The vertical axis given in the diagrams are the stress values (MPa), while the horizontal axis shows the strain values. 


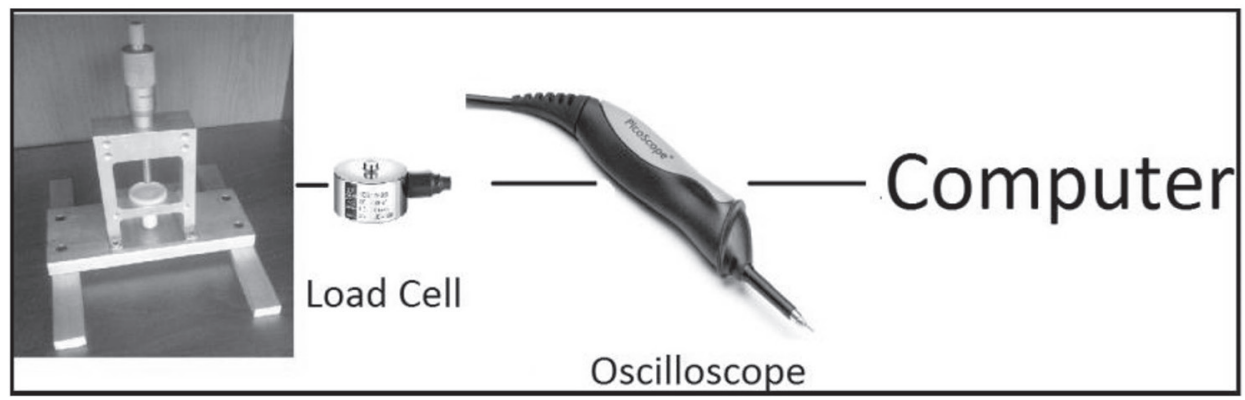

Fig. 1. Compression tests experimental setup for equilibrium modulus

\section{Compression tests for equilibrium modulus}

A stress relaxation test was performed using a cylindrical, plane-ended $3 \mathrm{~mm}$ diameter indenter positioned perpendicular to the superficial zone. Force and displacement were logged using Picolog (Pico Technology Ltd.). A $50 \mathrm{~kg}$ load cell (MNC-50L, CAS Corporation, South Korea) used to apply force. Initially, the force was applied and the cartilage was allowed to come to equilibrium. The cartilage surface was then displaced by $0.7 \mathrm{~mm}$ in total of ten steps; each step being $70 \mu \mathrm{m}$ [6]. Stress was calculated as load per unit macroscopic sectional field. Strain was calculated with reference to the relaxed length of the sample. The control and hydrogen peroxide exposed cartilage samples were immersed in a water bath filled with saline solution throughout testing under room temperature. Totally 12 cartilage samples (6 controls and 6 hydrogen peroxide exposed), were analyzed.

\section{Statistical analysis}

One-way analysis of variance (ANOVA) was used for statistical analysis. A p-value less than 0.05 was considered significant.

\section{RESULTS}

The slopes of the representative stress-strain curves show stiffness of the articular cartilage. Fig. 2 shows instantaneous stress-strain curves for control and hydrogen peroxide exposed experimental groups. The stiffness is higher in control 1 sample than control 2 according to slopes of the representative stress-strain curves (Fig. 2). It is obvious that slopes of control curves are higher than slopes of hydrogen peroxide curves. It means that hydrogen peroxide exposed experimental group has less stiffness than the control group. 


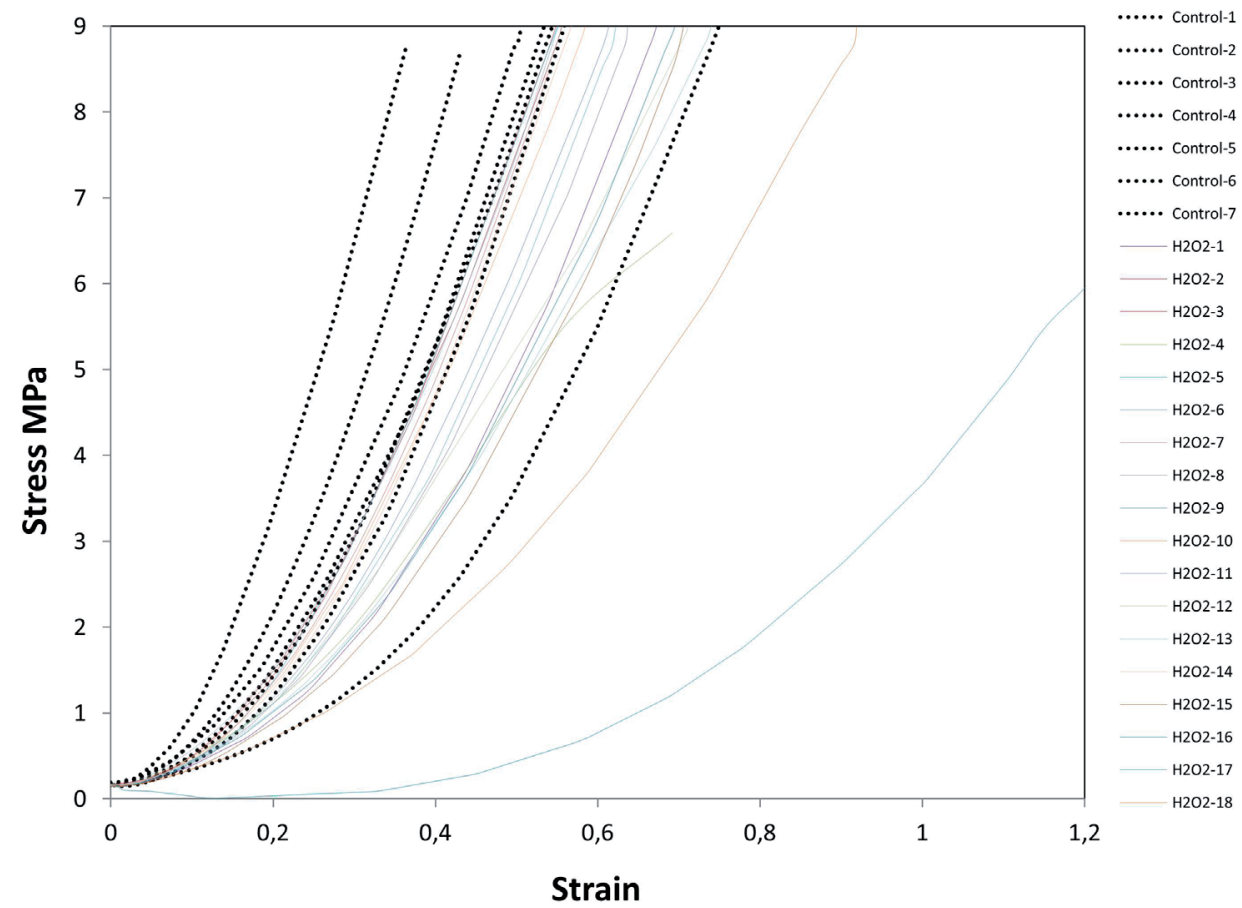

Fig. 2. Representative instantaneous stress-strain curves for articular cartilage ( 7 control and 18 hydrogen peroxide samples)

In instantaneous experiments, the maximum stress values are $8.28 \mathrm{MPa}$ (control 4) and $3.07 \mathrm{MPa}\left(\mathrm{H}_{2} \mathrm{O}_{2}-9\right)$, for control and hydrogen peroxide exposed experimental groups respectively at 0.35 strain. The lowest stress values are $1.72 \mathrm{MPa}$ (control 2) and $0.13 \mathrm{MPa}\left(\mathrm{H}_{2} \mathrm{O}_{2}-17\right)$, for control and hydrogen peroxide exposed experimental groups, respectively at 0.35 strain (Fig. 2).

Representative equilibrium stress-strain curves are shown for control and hydrogen peroxide exposed experimental groups in Fig. 3. The highest stress values are 10.80 $\mathrm{MPa}$ and 4.31 MPa for control and hydrogen peroxide exposed experimental group, respectively at 0.32 strain. The lowest values are $2.55 \mathrm{MPa}$ and $1.31 \mathrm{MPa}$ for control and hydrogen peroxide, respectively, at 0.32 strain. The relaxation time in control group is longer than experimental groups in our equilibrium experiments. The differences among control and experimental group stress increase after 0.25 strains (Fig. 3).

For the (0.33-0.35) strain, instantaneous compressive moduli values obtained from the stress-strain curves. These values are $22.79 \pm 3.18$ and $15.18 \pm 1.21$ for the control and hydrogen peroxide groups, respectively. The moduli values are clearly higher in the control as compared to the hydrogen peroxide exposed group (Fig. 4). These differences were statistically significant $(p=0.01)$.

For the $(0.24-0.32)$ strain, equilibrium compressive moduli values are obtained from the stress-strain curves. These values are $30.63 \pm 8.64$ and $11.98 \pm 2.49$ for the 


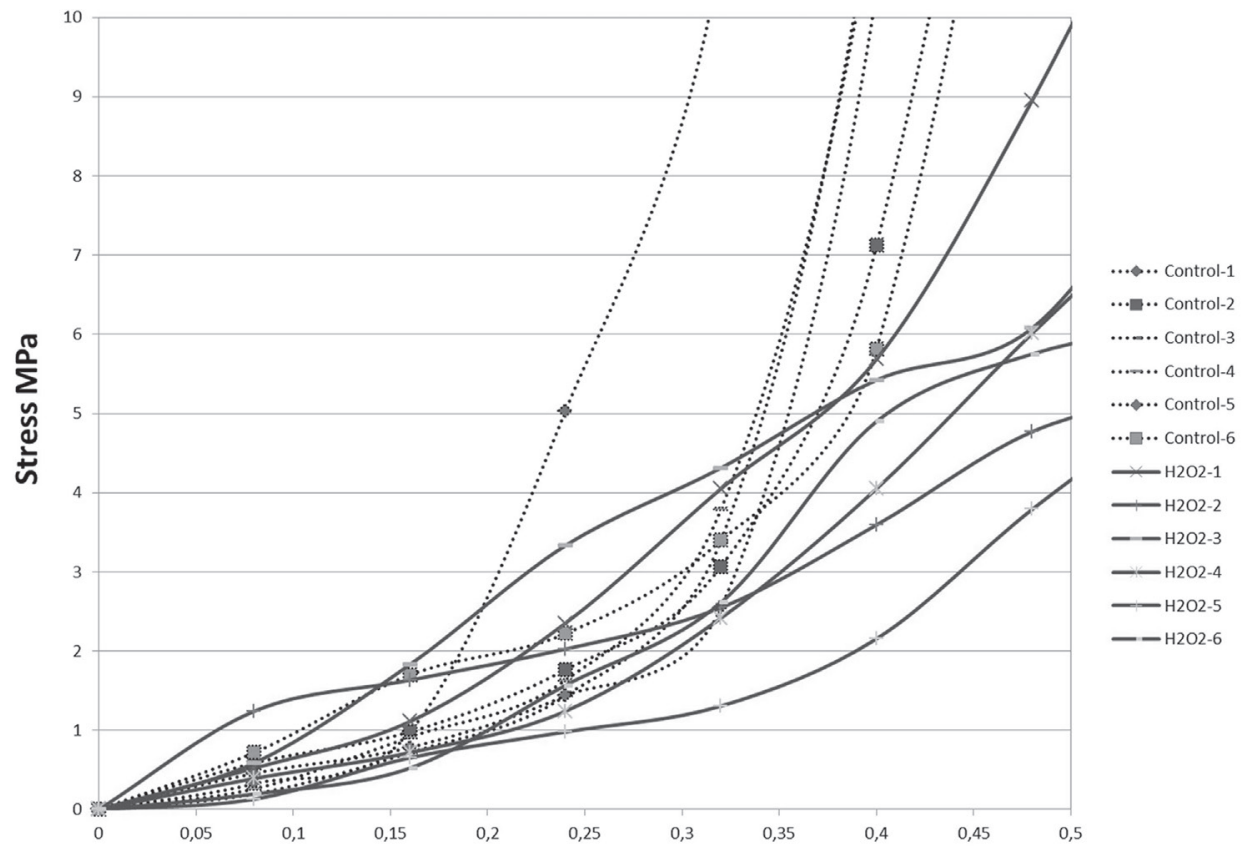

Strain

Fig. 3. Representative equilibrium stress-strain curves for articular cartilage (6 control and 6 hydrogen peroxide samples)

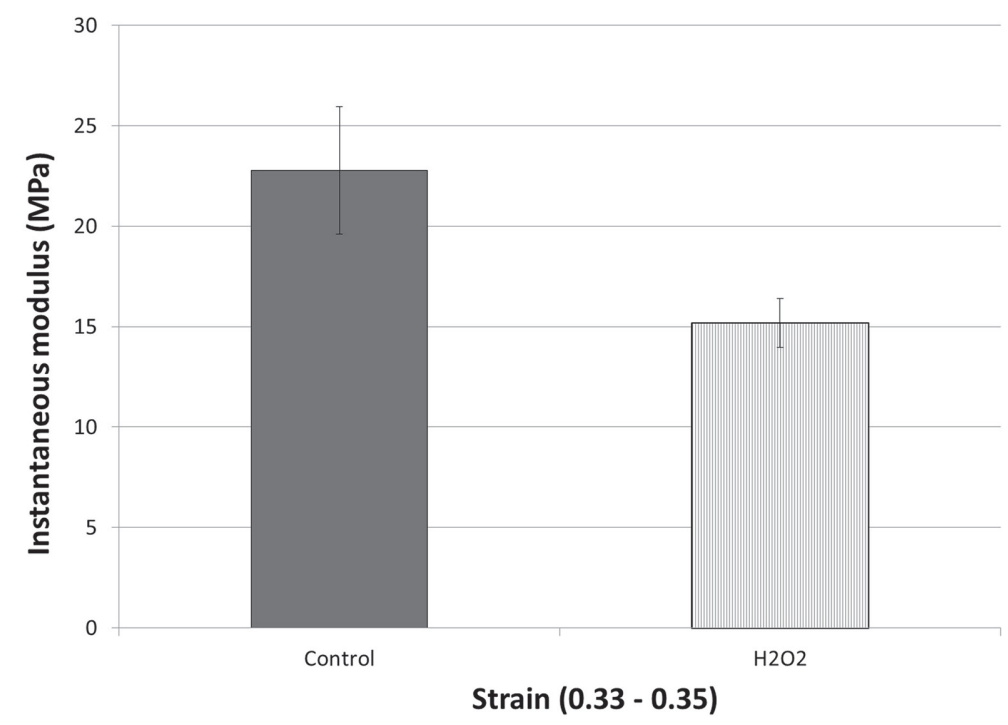

Fig. 4. Instantaneous modulus graph of the control and the experimental (hydrogen peroxide) groups obtained from Fig. 1 data 


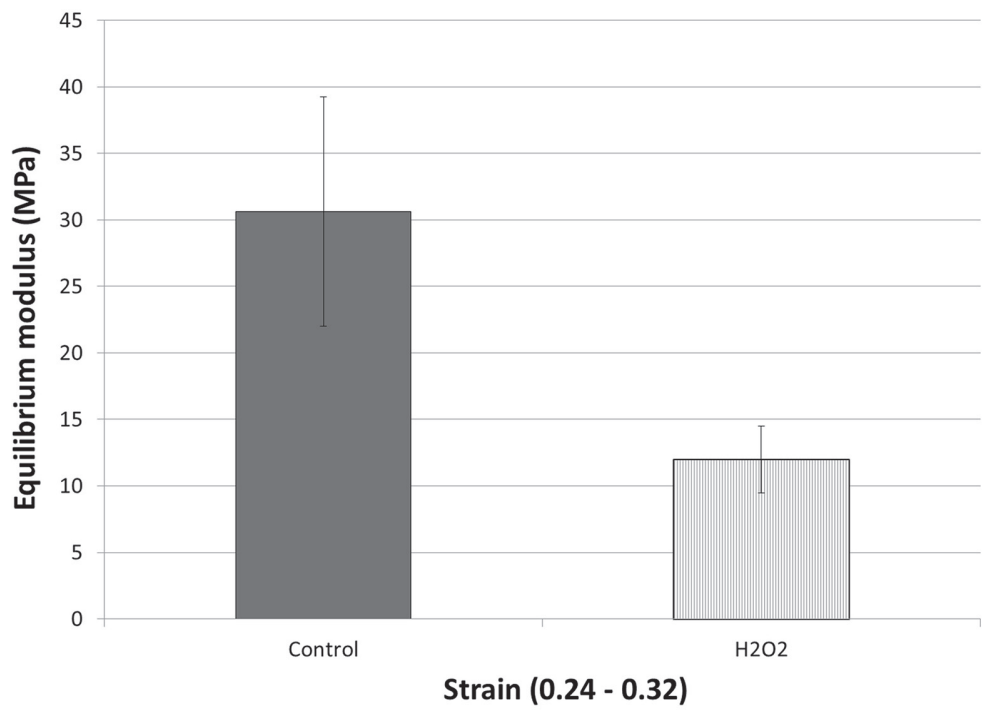

Fig. 5. Equilibrium modulus graph of the control and the experimental (hydrogen peroxide) groups obtained from Fig. 2 data

control and hydrogen peroxide groups, respectively. The moduli values higher in the control as compared to the hydrogen peroxide exposed group (Fig. 5). These differences were statistically insignificant ( $p=0.065$ ).

The moduli values of the control group were higher than that of hydrogen peroxide exposed experimental groups for both instantaneous experiments and equilibrium experiments. These results mean that the strength of the articular cartilage is negatively affected by the hydrogen peroxide.

\section{DISCUSSION}

ROS may randomly give damage to all matrix components, direct attack of proteoglycan and collagen molecules by free radicals is thought to cause the damage in cartilage matrix. Several in vitro studies have reported the degradation of cartilage tissue slices by increased ROS. The incubation of acid soluble type I collagen with superoxide anion radicals reduces collagen and averts the formation of fibrils by this collagen $[8,9,15]$.

Apart from the decrease of proteoglycan contents and down-regulation of Col2a1 and Agc1 genes in chondrocytes, deteriorating effects of hydrogen peroxide, a common form of ROS in extracellular matrix of cultured chondrocytes as reported [3]. Oxidative stress induced senescence in chondrocytes by hydrogen peroxide is characterized degradation of the extracellular matrix and a decrease in the synthesis of proteoglycan and type II collagen [5], it can further lead to apoptosis of chondrocytes 
and excessive production of various inflammatory cytokines, such as tumor necrosis factor-alpha, interleukin- 6 and interleukin-1beta, which further promote the expression of matrix metalloproteinases and other catabolic enzymes [23].

The collagen network is primarily responsible for instantaneous compressive stiffness of articular cartilage, while the proteoglycans are mainly responsible for the equilibrium compressive stiffness of cartilage [2, 12, 13]. In our instantaneous experiments for articular cartilage, stress-strain curves from the control and $\mathrm{H}_{2} \mathrm{O}_{2}$ groups resulted with the lowest stress values $1.72 \mathrm{MPa}$ and $0.13 \mathrm{MPa}$, respectively for 0.35 strains. Similarly, with the highest stress values $8.28 \mathrm{MPa}$ and $3.07 \mathrm{MPa}$ in the instantaneous stress-strain graph for control and experimental reduced stiffness in the exposed group is observed (see Fig 2). Differences between these groups were statistically significant $(p=0.01, p<0.05)$ (see Fig 4).

In our equilibrium experiments for the articular cartilage, as can be seen in Fig 3, the slopes of the representative stress-strain curves control group showed differences with that of the $\mathrm{H}_{2} \mathrm{O}_{2}$ exposed experimental group. Nevertheless, these differences amid groups were statistically insignificant $(p=0.065, p>0.05)$ (see Fig. 5). In both experiments, $\mathrm{H}_{2} \mathrm{O}_{2}$ treated samples show a considerably lower stress-strain curves with regard to the control. Although statistically insignificant, a deterioration of elements functioning in equilibrium tests, ie PGs, in $\mathrm{H}_{2} \mathrm{O}_{2}$ treated samples is indicated. In instantaneous experiments however, although responses are similar to the control, it is evidently seen that increasing stress values match with lower strain curves. In a previous study on the effects of ROS resulted by x-ray radiation on equilibrium stiffness of cartilage matrix [6], experimental curves were found to be more similar to those of the control indicating higher deteriorating rate of direct effect of ROS on the mechanical properties of the articular cartilage.

In conclusion, our results indicate that hydrogen peroxide induced oxidative damage causes reduction in the stiffness of the cartilage. In order to prevent such adverse effects of ROS induced by antiseptics like hydrogen peroxide, therapies increasing oxidant defenses are required $[3,9,14]$.

\section{ACKNOWLEDGMENTS}

This research has been supported by Mehmet Akif Ersoy University, Scientific Research Fund, Project Number: 333-NAP-16.

\section{REFERENCES}

1. Atiyeh, B. S., Dibo, S. A., Hayek, S. N. (2009) Wound cleansing, topical antiseptics and wound healing. Int. Wound J. 6, 420-430.

2. Bader, D. L., Kempson, G. E., Egan, J., Gilbey, W., Barrett, A. J. (1992) The effects of selective matrix degradation on the short-term compressive properties of adult human articular cartilage. Biochim. Biophys. Acta. 1116, 147-154.

3. Bhatti, F. U. R., Mehmood, A., Wajid, N., Rauf, M., Khan, S. N., Riazuddin, S. (2013) Vitamin e protects chondrocytes against hydrogen peroxide-induced oxidative stress in vitro. Inflamm. Res. 62, 781-789. 
4. Bonifacio, A., Beleites, C., Vittur, F., Marsich, E., Semeraro, S., Paoletti, S., Sergoa, V. (2010) Chemical imaging of articular cartilage sections with Raman mapping, employing uni- and multivariate methods for data analysis. Analyst 135, 3193-3204.

5. Brandl, A., Hartmann, A., Bechmann, V., Graf, B., Nerlich, M., Angele, P. (2011) Oxidative stress induces senescence in chondrocytes. J. Orthop. Res. 29, 1114-1120.

6. Cicek, E. (2016) Effect of X-ray irradiation on articular cartilage mechanical properties. Acta Phys. Pol. A 129, 200-202.

7. Drosou, A., Falabella, A., Kirsner, R. (2003) Antiseptics on wounds: an area of controversy. Wounds $15,149-166$.

8. Greenwald, R. A., Moy, W. W. (1979) Inhibition of collagen gelation by action of the superoxide radical. Arthritis Rheum. 22, 251-259.

9. Henrotin, Y. E., Bruckner, P., Pujol, J. P. L. (2003) The role of reactive oxygen species in homeostasis and degradation of cartilage. Osteoarthr. Cartil. 11, 747-755.

10. Huber, M., Trattnig, S., Lintner, F. (2000) Anatomy, Biochemistry, and Physiology of Articular Cartilage. Invest. Radiol. 35, 573-580.

11. Julkunen, P., Wilson, W., Jurvelin, J. S., Rieppo, J., Qu, C. J., Lammi, M. J., Korhonen, R. K. (2008) Stress-relaxation of human patellar articular cartilage in unconfined compression: Prediction of mechanical response by tissue composition and structure. J. Biomech. 41, 1978-1986.

12. Korhonen, R. K., Laasanen, M. S., Toyras, J., Lappalainen, R., Helminen, H. J., Jurvelin, J. S. (2003) Fibril reinforced poroelastic model predicts specifically mechanical behavior of normal, proteoglycan depleted and collagen degraded articular cartilage. J. Biomech. 36, 1373-1379.

13. Laasanen, M. S., Töyräs, J., Korhonen, R. K., Rieppo, J., Saarakkala, S., Nieminen, M., Hirvonen, J., Jurvelin, J. (2003) Biomechanical properties of knee articular cartilage. Biorheology 40, 133-140.

14. Lean, J., Davies, J., Fuller, K., Jagger, C., Kirstein, B., Partington, G., Urry, Z., Chambers, T. (2003) A crucial role for thiol antioxidants in estrogen-deficiency bone loss. J. Clin. Invest. 112, 915-923.

15. Monboisse, J. C., Braquet, P., Randoux, A., Borel, J. P. (1983) Non-enzymatic degradation of acidsoluble calf skin collagen by superoxide ion: protective effect of flavonoids. Biochem. Pharmacol. 32, $53-58$.

16. Mow, V. C., Ratcliffe, A., Poole, A. R. (2000) Cartilage and diarthrodial joints as paradigms for hierarchical materials and structures. Biomaterials 13, 67-97.

17. Na, J. Y., Song, K., Kim, S., Kwon, J. (2016) Rutin protects rat articular chondrocytes against oxidative stress induced by hydrogen peroxide through SIRT1 activation. Biochem. Biophys. Res. Commun. 473, 1301-1308.

18. Omata, S., Sawae, Y., Murakami, T. (2012) Tissue Development and Mechanical Property in the Regenerated-Cartilage Tissue. In: Ceccherini-Nelli, L., Matteoli, B. (ed.) Biomedical Tissue Culture. InTech, Italy, pp. 1-8.

19. Ouyang, X., Wei, B., Hong, S. D., Wang, J. R., Xin, F., Wang, L., Yang, X. W., Wang, L. M. (2015) Study on the Mechanisms of Cartilage Tissue Damage Caused by Hydrogen Peroxide. Cell Biochem. Biophys. 72, 343-348

20. Röhner, E., Kolar, P., Seeger, J. B., Arnholdt, J., Thiele, K., Perka, C., Matziolis, G. (2011) Toxicity of antiseptics against chondrocytes: What is best for the cartilage in septic joint surgery? Int. Orthop. $35,1719-1723$.

21. Schaumburger, J., Beckmann, J., Springorum. H., Handel, M., Anders, S., Kalteis, T., Grifka, J., Rath, B. (2010) Toxizität lokaler Antiseptika auf Chondrozyten in vitro. Z. Orthop. Unfall. 148, 39-43.

22. Young, I. C., Chuang, S. T., Hsu, C. H., Sun, Y. J., Lin, F. H. (2016) C-phycocyanin alleviates osteoarthritic injury in chondrocytes stimulated with $\mathrm{H}_{2} \mathrm{O}_{2}$ and compressive stress. Int. J. Biol. Macromol. 93, 852-859.

23. Zhuang, C., Xu, N. W., Gao, G. M., Ni, S., Miao, K. S., Li, C. K., Wang, L. M., Xie, H. G. (2016) Polysaccharide from Angelica sinensis protects chondrocytes from $\mathrm{H}_{2} \mathrm{O}_{2}$-induced apoptosis through its antioxidant effects in vitro. Int. J. Biol. Macromol. 87, 322-328. 